\title{
The "beam of darkness": Spreading of the attentional blink within and between objects
}

\author{
Markus Conci and Hermann J. Müller \\ Ludwig-Maximilians Universität München, Munich, Germany
}

\begin{abstract}
When two targets (T1 and T2) are inserted into a rapid serial visual presentation (RSVP) stream of nontargets, observers are impaired at identifying T2 when it is presented within half a second after T1. This transient drop in performance, or attentional blink $(\mathrm{AB})$, has been attributed to a temporary unavailability of task-critical processing resources. In the present study, we investigated how object-based attention modulates the AB, by presenting four synchronized RSVP streams in the corners of two rectangular bars (e.g., one above and one below fixation). The results from four experiments revealed that the $\mathrm{AB}$ increased within short temporal lags (of up to $\sim 400 \mathrm{msec}$ ) when T2 was presented on the same, rather than a different, bar as T1 (with T1-T2 spatial distance controlled for). Thus, the $\mathrm{AB}$ is seen to spread across entire object groupings, suggesting that the spatiotemporal resolution of attention is modulated by global-object information.
\end{abstract}

Complex, natural environments challenge the visual system in the processing of multiple pieces of information arriving simultaneously from different ambient sources. However, often, even salient events go unnoticed, because of limitations in how much information the visual system can process at a given point in time. Thus, for example, awareness of a given (target) stimulus may be substantially impaired if attention is distracted by a competing stimulus or engaged elsewhere (see, e.g., Kim \& Blake, 2005, for a review).

The attentional blink (AB) phenomenon provides a prominent example of such a bottleneck in the processing of multiple stimuli (e.g., Chun \& Potter, 1995; Raymond, Shapiro, \& Arnell, 1992). In a typical AB experiment, observers are presented with two targets (T1 and T2) embedded within a rapid serial visual presentation (RSVP) stream of nontargets. When the temporal interval between the two targets is less than $\sim 500 \mathrm{msec}$, observers frequently fail to identify $\mathrm{T} 2$, whereas they show good performance in identifying T1. In general, this pattern of performance shows that at least some aspects of visual processing are confined to only one object at a time (but see below). The reduction of T2 identification at relatively short temporal lags occurs because limited attentional resources are still engaged in processing and consolidating T1. At longer lags, the ability to process $\mathrm{T} 2$ recovers because resources are released when T1 processing has terminated (for reviews, see Hommel et al., 2006, and Shapiro, Raymond, \& Arnell, 1997).

Despite monotonic increases in T2 identification with longer T1-T2 lags, many studies have reported the $\mathrm{AB}$ effect to be characterized by a U-shaped function, with relatively unimpaired performance if $\mathrm{T} 2$ is presented directly after $\mathrm{T} 1$ and a transitory drop in accuracy only thereafter (e.g., Chun \& Potter, 1995; Raymond et al., 1992). This effect of lag 1 sparing has been interpreted in terms of the visual system's being able to process the two targets together (in a batch) as long as they appear in direct temporal succession. However, in a meta-analysis of $\mathrm{AB}$ experiments, lag 1 sparing has been revealed to occur only in cases in which no attentional switch (between locations or categories) was required between targets (Visser, Bischof, \& Di Lollo, 1999; see also Juola, Botella, \& Palacios, 2004).

Studies of the AB have primarily investigated the temporal modulation of the attentional engagement, with a stream of stimuli presented at a single spatial location. However, in some studies, attempts have also been made to determine the spatiotemporal characteristics of the AB. Presenting observers with two noncontiguous RSVP streams, Kawahara and Yamada (2006; Yamada \& Kawahara, 2007) found, with two T1 and T2 targets that appeared simultaneously at two adjacent spatial positions, that the visual system can monitor multiple concurrent streams. However, if targets switch unpredictably from one to the other of two spatially segregated streams, shifts of attention lead to an increase in the AB at short T1-T2 lags (Juola et al., 2004; Shih, 2000; Visser, Zuvic, Bischof, \& Di Lollo, 1999). Indeed, in such situations, if observers do not allocate attention broadly to multiple streams (e.g., if they focus narrowly on a T1 location known in 
advance), the AB is especially pronounced at lag 1 (Jefferies, Ghorashi, Kawahara, \& Di Lollo, 2007).

In a more systematic investigation of the relation between spatial and temporal parameters, Kristjánsson and Nakayama (2002) presented observers with six synchronized RSVP streams, arranged in a circle around central fixation, and required them to identify two targets presented at distinct spatial and temporal positions. The results showed that recovery from the $A B$ increased with larger temporal lags and with increased spatial separation between the two targets. Kristjánsson and Nakayama took this pattern to be indicative of a gradient of attentional resolution that depends on both space and time, with spatial and temporal selection being mediated by a common attentional mechanism: Both spatial and temporal parameters determine the ease with which attention can be reallocated from a given T1 stimulus to another T2 stimulus in a dynamically changing environment (see Jiang \& Chun, 2001, for a similar proposal).

\section{Object-Based Attention}

A large body of evidence suggests that attentional selection is not based simply on unparsed regions of visual space but is determined by representations of integrated, or grouped, "objects" within the field (e.g., Driver \& Baylis, 1989; Duncan, 1984; Egly, Driver, \& Rafal, 1994; Lamy \& Egeth, 2002; O'Grady \& Müller, 2000; Vecera \& Farah, 1994; Watson \& Kramer, 1999). For instance, Egly et al. asked their observers to detect a luminance change (target) at one of four ends of two bars (e.g., two vertical bars to the left and right of central fixation). On a given trial, one end of a bar was precued (i.e., brightened), with the target appearing at this location on $75 \%$ of the trials (valid condition). If the target appeared at an invalid (i.e., uncued) position, performance costs were greater when this position was located on the other bar, rather than on the same bar as the cue (with the same cue-target distance in both conditions). This finding indicates that objects modulate how attention is engaged, with reallocation of attention from the cued to the target position operating more efficiently within objects, rather than between objects.

Follow-up studies revealed an influence of the hierarchical level of perceptual grouping on object-based effects. In particular, Watson and Kramer (1999) showed that object-based effects are obtained when same-object targets belong to the same uniformly connected region, but not when they belong to separate regions grouped into a higher order structure on the basis of Gestalt principles (see Palmer \& Rock, 1994, for the distinction between single uniformly connected and multiple grouped regions). For such higher order objects, object-based effects are observed only when the task requires spatial shifts of attention (Lamy \& Egeth, 2002).

In line with object-based attention, Raymond (2003; see also Kellie \& Shapiro, 2004) showed (for a single, uniformly connected object) that the $\mathrm{AB}$ is associated primarily with creating whole-object representations, rather than registering component features. In the experi- ment, observers were instructed to report two target features (T1 and T2), a thickened line and an accessory line segment, embedded within an RSVP stream of rotated trident-shaped filler objects. The target features could be presented either on the same type of object (two tridents) or on different types (a trident and an arrowhead). If the target features were presented on the same type of object, the $\mathrm{AB}$ was markedly reduced, as compared with when the same features were presented on different types. Raymond suggested that the object file (i.e., the internal object description) created for identification of $\mathrm{T} 1$ could be updated easily when $\mathrm{T} 2$ was subsequently presented on the same type of object. However, if T2 is presented on a different type of object, a new object file would have to be created, leading to a more marked AB. Consequently, the $\mathrm{AB}$ appears to reflect limitations in creating new object representations, rather than in encoding simple features.

The aim of the present study was to investigate whether and how the processing of two targets presented at different positions in space and time would be modulated by object groupings-in particular, global groupings based on common region (see Palmer, 1992), instead of the single, uniformly connected objects used by Raymond (2003). As has been indicated by previous studies (Lamy \& Egeth, 2002; Watson \& Kramer, 1999), both uniformly connected objects and higher order object groupings may differ substantially in the extent to which they engage object-based attention. Consequently, to examine the role of higher order object groupings in the $\mathrm{AB}$, we implemented an RSVP variant of the spatial-cuing paradigm introduced by Egly et al. (1994): Observers were presented with two rectangular (bar) objects (e.g., one left and one right of fixation), with an RSVP stream displayed at each end of each bar (i.e., 2 bars $\times 2$ ends $=$ 4 streams in total). Observers were required to identify two uniquely colored target letters, either from the RSVP streams within the same bar or from streams on different bars (see Figure 1). The crucial comparison involved the identification of target letters that were inserted into two neighboring streams, either on the same bar or on different bars, with the same T1-T2 spatial separation in both conditions. Consequently, by systematically varying the T1-T2 lag and whether T2 was presented on the same or a different bar (while controlling for the spatial distance), we investigated whether object-based attention would modulate the $\mathrm{AB}$.

Extrapolating from Egly et al. (1994), object-based influences on the spatiotemporal distribution of attention could be explained by a cost of shifting attention between global objects. Thus, T2 should be processed more efficiently if it is located on the same object as T1 (rather than on the other object); that is, T1 would act as a spatial-object cue, facilitating redirection of spatial attention within the cued bar object. On the basis of these considerations, we initially expected to find a reduced $\mathrm{AB}$ when the T2 was located within the same global object as $\mathrm{T} 1$ and, conversely, an increased $\mathrm{AB}$ when both targets were located within distinct, global objects. 


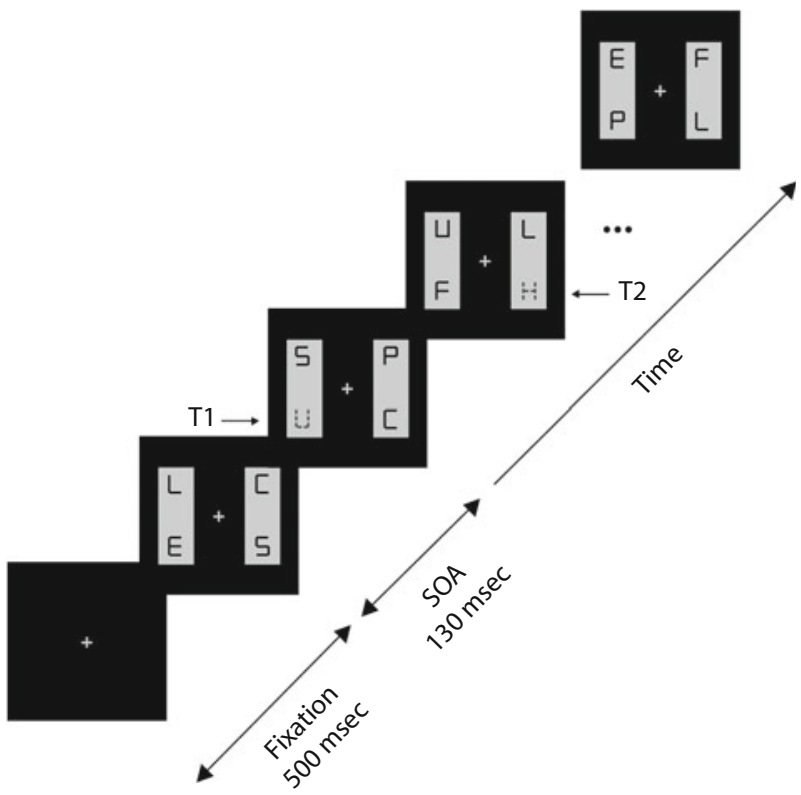

Figure 1. Schematic example of the rapid serial visual presentation sequences in Experiment 1. Each trial started with a fixation cross presented for $500 \mathrm{msec}$, followed by a sequence of 16 displays each presented with a stimulus onset asynchrony (SOA) of 130 msec. Displays contained two gray bars oriented either vertically (as in the example sequence) or horizontally and four letters that served as either nontargets (solid) or targets (dashed; T1 and T2). Targets were presented in red, and nontargets in black.

\section{EXPERIMENT 1}

Experiment 1 was performed to investigate objectbased effects on the AB. We employed an RSVP variant of the spatial-cuing paradigm by Egly et al. (1994). In this variant of the paradigm, observers were presented with two rectangular objects, with an RSVP stream displayed at each end of each bar (see Figure 1). Two uniquely colored target letters were presented in a sequence (with variable lag), either from the RSVP streams within the same bar or from streams on different bars (at equal spatial distances). Thus, this approach allowed assessing whether the rectangular object groupings can influence the AB.

\section{Method}

Participants. Ten paid observers (five of them male; mean age $=$ 27.1 years; normal or corrected-to-normal visual acuity) participated in the experiment.

Apparatus and Stimuli. The experiment was controlled by an IBM PC-compatible computer using MATLAB routines and Psychophysics Toolbox extensions (Brainard, 1997; Pelli, 1997), and the stimuli were presented on a 17 -in. monitor $(1,024 \times 768$ pixel screen resolution, $85-\mathrm{Hz}$ refresh rate). On each trial, two gray filledin bars (luminance: $3.81 \mathrm{~cd} / \mathrm{m}^{2}$ ) were presented on a black background $\left(0.01 \mathrm{~cd} / \mathrm{m}^{2}\right)$. The bars subtended $2.3^{\circ} \times 9.9^{\circ}$ of visual angle and were displayed in either vertical or horizontal orientation, shifted by $3.6^{\circ}$ from the central white fixation cross (stimulus sizes were comparable to those in Egly et al., 1994, and Lamy \& Egeth, 2002). At the ends of the two bars, four synchronized RSVP streams of letters were presented at a distance of $5^{\circ}$ from the fixation cross. Within each stream, eight different uppercase letters were presented $(H, U$,
E, P, S, C, F, L) that subtended $1^{\circ} \times 1.5^{\circ}$, composed of horizontal and vertical lines (line thickness, 1 pixel). Two targets were randomly chosen from the set of letters and were presented in red (luminance: $2.72 \mathrm{~cd} / \mathrm{m}^{2}$ ) at two neighboring RSVP streams (T2 never appeared at the same locations as T1 or on the location diagonally opposite to $\mathrm{T} 1$ ). Nontargets were displayed in black (luminance: $0.01 \mathrm{~cd} / \mathrm{m}^{2}$ ).

Procedure and Design. Each trial started with the presentation of a central fixation cross that remained on the screen for the complete trial. After $500 \mathrm{msec}$, the two bars were presented at either a horizontal or a vertical orientation, with synchronized RSVP streams displayed at the four ends of the bars. Each stream presented a randomized sequence of 16 letters, with each of the 8 possible letters presented only twice within a given stream. Every letter was presented for $110 \mathrm{msec}$, followed by a blank interval of $20 \mathrm{msec}$, resulting in a stimulus onset asynchrony (SOA) of $130 \mathrm{msec}$. The SOA duration was determined in a pilot experiment (which showed that observers had great difficulty performing the task at shorter durations) and was comparable to the durations in previous multipleRSVP stream experiments (100- to 140-msec SOAs; see Kawahara \& Yamada, 2006; Kristjánsson \& Nakayama, 2002). On a given trial, two red target letters were presented. The first target (T1) was randomly assigned to one of seven temporal serial positions from 3 through 9 within the stream of 16 letters. The second target (T2) was presented at a (horizontally or vertically) neighboring RSVP stream at one of five different temporal lags $(130,260,390,520$, or $650 \mathrm{msec}$ ). RSVP stream distractors continued to be presented during the lag. After the presentation of the RSVP streams, the displays were removed from the screen, and the observers were asked to identify the two targets by typing the corresponding keys in the correct order. The observers were instructed to respond as accurately as possible, with particular emphasis on $\mathrm{T} 1$ accuracy in order to maximize the number of trials for the analysis of T2 accuracy. In case of an erroneous T1 response, visual feedback was provided in the form of a "-" sign presented for $500 \mathrm{msec}$. Trials were separated from one another by an interval of $1,000 \mathrm{msec}$.

The experiment systematically varied three factors: bar orientation (horizontal vs. vertical), T2 location (on same vs. different bar, relative to T1), and $T 1-T 2 \operatorname{lag}(130,260,390,520$, or $650 \mathrm{msec})$, with all possible factorial combinations presented in random order. In addition, there were equal numbers of left-right (top-bottom) and right-left (bottom-top) target sequences, respectively, to control for possible systematic influences of the succession of T1 and T2 locations. Each observer completed two sessions, each of 40 practice plus 400 experimental trials. Sessions were divided into 10 blocks of 40 trials each.

\section{Results}

Accuracy of T1 identifications was relatively high, with an average of $75 \%$ of correct responses across all conditions. T1 accuracy was influenced by bar orientation: Correct identifications were more frequent with vertical than with horizontal bars $[77 \%$ vs. $73 \% ; t(9)=4.17, p<$ $.003]$.

Of prime interest for the purpose of the experiment is the accuracy of T2 identification. Estimates of T2 accuracy were based only on trials on which $\mathrm{T} 1$ had been identified correctly. This approach is commonly taken in $\mathrm{AB}$ experiments because, for incorrect $\mathrm{T} 1$ responses, the cause of the error is not known, making it hard to determine its effect on the processing of T2. Figure 2 presents T2 accuracy as a function of lag, separately for the sameand different-object conditions. A three-way repeated measures ANOVA of correct T2 responses, with main terms for bar orientation (horizontal, vertical), T2 location (same object, different object), and T1-T2 lag (130, 


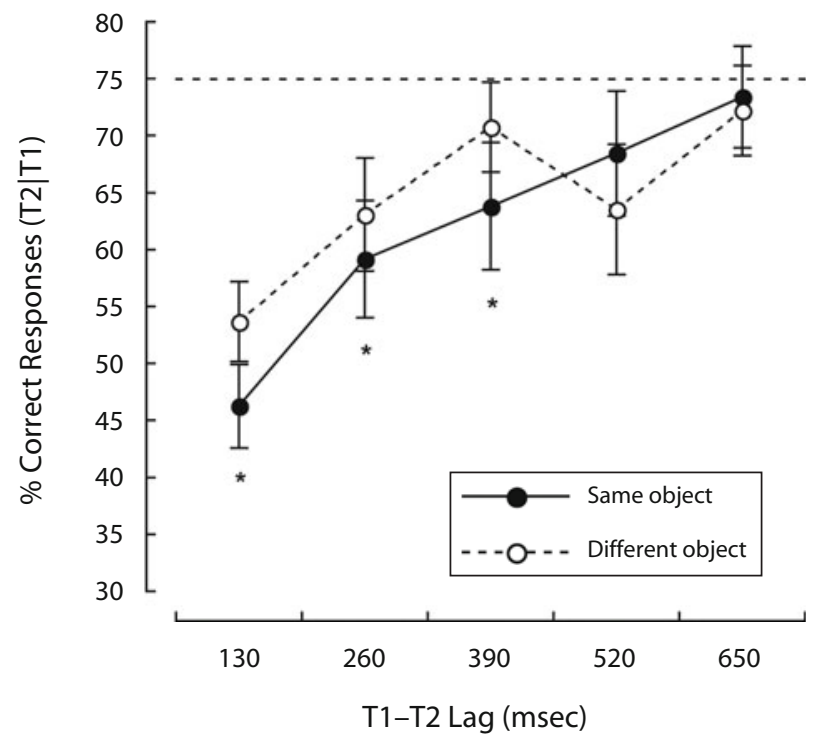

Figure 2. The results of Experiment 1: Mean percentage of correct identifications of the second target (T2), given that the first target (T1) had been identified correctly. Correct identifications are presented as a function of the temporal lag from the onset of T1 to the onset of T2, separately for same-object (solid line) and different-object (dashed line) conditions, with significant differences between same- and different-object conditions indicated by an asterisk. In addition, the dashed horizontal line indicates the level of overall T1 accuracy. Error bars indicate the standard errors of the means.

$260,390,520,650 \mathrm{msec})$, revealed all main effects to be significant: bar orientation $[F(1,9)=19.97, p<.003]$, $\mathrm{T} 2$ location $[F(1,9)=5.31, p<.05]$, and lag $[F(4,36)=$ $18.01, p<.001]$. T2 accuracy was higher for vertical than for horizontal bar orientations ( $65 \%$ vs. $62 \%$ ), mirroring the pattern observed for T1. In addition, performance was higher for different- than for same-object T2 locations ( $65 \%$ vs. $62 \%$ ). Finally, T2 accuracy increased reasonably linearly with T1-T2 lag $(50 \%, 61 \%, 67 \%, 66 \%$, and $72 \%$ for lags of 130, 260, 390, 520, and $650 \mathrm{msec}$, respectively; $\left.R^{2}=.86, p<.03\right)$.

Moreover, there were several significant interactions. Theoretically of most importance is that of $\mathrm{T} 2$ location with lag $[F(4,36)=3.88, p<.02]$, which was due to significant same-object costs for the first three temporal lags (see Figure 2): When T2 was presented at a lag of 130,260 , or $390 \mathrm{msec}, \mathrm{T} 2$ identification was less accurate when it was presented on the same, rather than on a different, object as T1 (same-object costs were $7 \%, 4 \%$, and $7 \%$ for lags of 130, 260, and $390 \mathrm{msec}$, respectively; all $p$ s $<$ $.04)$; for lags longer than $390 \mathrm{msec}$, this difference was no longer evident (all $p \mathrm{~s}>.11$ ).

In addition, the T2 location $\times$ bar orientation $[F(1,9)=$ $4.59, p=.06$ ] and T2 location $\times$ bar orientation $\times$ lag $[F(4,36)=3.06, p<.03]$ interactions were (marginally) significant. The same-object costs tended to be larger, overall, for vertical than for horizontal bar configurations (costs of $6 \%$ and $1 \%$, respectively). However, the significant three-way interaction revealed that this general effect resulted from the lag of $650 \mathrm{msec}[t(9)=3.6, p<.007]$, rather than from shorter lags (all $p \mathrm{~s}>.3$; see Table 1): Whereas the 650-msec lag led to a substantial same-object cost for the vertical configuration, the reverse-namely, a same-object benefit-was observable for horizontal configurations. At all shorter lags, there were no statistically reliable differences between vertical and horizontal configurations. This pattern suggests that for T1-T2 lags shorter than $650 \mathrm{msec}$, object information had an influence on T2 accuracy. However, at a lag of $650 \mathrm{msec}$, this influence was no longer evident, since it was replaced by a preference for horizontal over vertical scanning directions.

A follow-up analysis examined whether same-object costs (at lags of $<650 \mathrm{msec}$ ) would also be evident when T1 was missed. A two-way repeated measures ANOVA of correct $\mathrm{T} 2$ responses (given an incorrect $\mathrm{T} 1$ response), with main terms for T2 location (same object, different object) and lag $(130,260,390,520,650 \mathrm{msec})$, revealed the main effect of lag $[F(4,36)=5.09, p<.003]$ and the interaction of lag with T2 location $[F(4,36)=8.63, p<$ $.001]$ to be significant. As with correct T1 identifications, there was an increase in T2 accuracy as the lag increased $(43 \%, 44 \%, 52 \%, 58 \%$, and $64 \%$ for lags of $130,260,390$, 520 , and $650 \mathrm{msec}$, respectively). In addition, there were reliable same-object costs, of $13 \%$ and $25 \%$, for lags of 260 and $390 \mathrm{msec}$, respectively (both $p \mathrm{~s}<.004$ ).

\section{Discussion}

The results from Experiment 1 confirm that globalobject information can modulate the spread of the AB: T2 identification accuracy was lower when T2 was presented on the same bar as T1, as compared with a different bar (at identical T1-T2 spatial distances), and this effect was especially pronounced for short temporal lags. This pattern was the same whether T1 identification was correct or incorrect. Thus, if the second target followed the first within $400 \mathrm{msec}$, a significant object-based modulation of performance was obtained, with same-object costs of some $6 \%$ when $\mathrm{T} 1$ identification was correct. At lags longer than $400 \mathrm{msec}$, the difference between same and different objects vanished (with a statistically unreliable same-object benefit of 3\% at these lags). Thus, these results show that although there was a clear effect of the global objects on the $\mathrm{AB}$, its direction was exactly opposite to the effect that would have been expected on the basis of the findings from spatial-cuing studies (i.e., costs rather than benefits, as in Egly et al., 1994). Rather, the reduction in identification accuracy for $\mathrm{T} 2$ at short temporal lags implies that the

Table 1

Same-Object Costs (in Percentages) in Experiment 1 for Vertical and Horizontal Bar Orientations (and Corresponding Relative Differences Between Display Configurations) at Varying Temporal Lags

\begin{tabular}{lccccc}
\hline & \multicolumn{5}{c}{ Lag (msec) } \\
\cline { 2 - 6 } \multicolumn{1}{c}{ Bar Orientation } & 130 & 260 & 390 & \multicolumn{1}{c}{520} & \multicolumn{1}{c}{650} \\
\hline Vertical & 6.8 & 6.6 & 9.1 & -4.8 & 11.2 \\
Horizontal & 7.9 & 1.3 & 4.8 & -5.0 & -13.6 \\
Relative difference & 1.1 & 5.3 & 4.3 & 0.2 & $24.8^{*}$ \\
\hline
\end{tabular}

Note-Same-object benefits are indexed by a negative value. $\quad$ *Significant difference at $p<.007$. 
$\mathrm{AB}$ has a stronger effect on neighboring locations within the same (as opposed to a different) object.

Note that there was no indication of lag 1 sparing in Experiment 1. This is in line with previous studies on the spatiotemporal dynamics of the AB, which showed that, with T1 and T2 presented in different RSVP streams, there is a need to shift attention from one to the other location, eliminating lag 1 sparing (Juola et al., 2004; Kristjánsson \& Nakayama, 2002; Shih, 2000; Visser, Bischof, \& Di Lollo, 1999; Visser, Zuvic, et al., 1999).

Next, in Experiment 2, we set out to replicate the objectbased costs in the $\mathrm{AB}$ effect in a variant of the paradigm that permitted T2 to appear at any of the four possible stimulus locations.

\section{EXPERIMENT 2}

Experiment 1 demonstrated an object-based influence on the AB. However, one potential confound was that the sequence of the two sequential targets was not randomized: T2 was always presented at a neighboring spatial location, relative to $\mathrm{T} 1$, and this might have biased the distribution of attention on a given trial. In order to rule out the possibility that the unequal distribution of T2 locations had an influence on our results, in Experiment 2, $\mathrm{T} 2$ could occur in any of the four possible locations with equal probability. Thus, T2 could now be presented on the same position as $\mathrm{T} 1$, on a neighboring position (within the same or different objects) as $\mathrm{T} 1$, or on the position diagonally opposite to T1.

\section{Method}

Experiment 2 was identical to the previous experiment, except that T2 could now occur at all four possible RSVP stream locations with equal probability: $\mathrm{T} 2$ could be presented at the same position as $\mathrm{T} 1$, at the neighboring position within the same or a different object as T1, or at the position diagonally opposite to T1. In Experiment 2, only vertical bar orientations were presented (see Figure 1), since the orientation of the bars was not found to be critical for objectbased $\mathrm{AB}$ effects in Experiment 1. Ten paid observers (4 of them male; mean age $=25.9$ years; normal or corrected-to-normal visual acuity) participated in the experiment. In summary, the experiment varied the factors of $T 2$ location (same position, same object, different object, or different [object] diagonal) and T1-T2 lag (130, 260, 390,520 , or $650 \mathrm{msec}$ ), with all possible factorial combinations presented in random order. Each observer completed two sessions, each of 40 practice plus 400 experimental trials (divided into 10 blocks).

\section{Results}

Accuracy of T1 identifications was again relatively high, with an average of $77 \%$ of correct responses, comparable to the level of correct identifications in Experiment 1.

For the analysis of T2 responses (given a correct T1 response), the same- and different-object conditions were compared, since these were equal with regard to the spatial distances between the two targets. Figure 3A presents T2 accuracy as a function of lag, separately for the sameand different-object conditions. A two-way repeated measures ANOVA of correct T2 responses, with main terms for T2 location (same object, different object) and T1-T2 lag $(130,260,390,520,650 \mathrm{msec})$, revealed both main effects and their interaction to be significant [T2 location,
$F(1,9)=9.55, p<.02$; lag, $F(4,36)=10.26, p<.001$; T2 location $\times$ lag, $F(4,36)=3.01, p<.04]$. Accuracy was higher for different- than for same-object T2 locations (69\% vs. 61\%), and performance showed an increase over the first three lags $(51 \%, 65 \%, 71 \%, 70 \%$, and $68 \%$ for lags of $130,260,390,520$, and $650 \mathrm{msec}$, respectively). In addition, the significant interaction indicated that there were reliable same-object costs of some $14 \%$ at temporal lags shorter than $400 \mathrm{msec}$ (and a same-object benefit of $6 \%$ at the 650 -msec lag; all $p \mathrm{~s}<.04)$. Thus, this pattern of results mirrors the findings from Experiment 1.

Next, to compare T2 accuracy for RSVP stream locations within the same object, a further repeated measures ANOVA of correct T2 responses was performed, with main terms for T2 location (same position, same object) and T1-T2 lag. This analysis revealed only a significant main effect of lag $[F(4,36)=14.05, p<.001]$, due to an increase in T2 accuracy with an increasing temporal separation between the two targets (from $48 \%$ to $73 \%$ for lags from 130 to $650 \mathrm{msec}$ ). However, T2 accuracy did not differ significantly, at any lag, between positions within the same object (all $p \mathrm{~s}>.35$; see Figure 3B).

Finally, T2 accuracy was compared for the two RSVP stream locations within the different object (relative to T1). A repeated measures ANOVA of correct T2 responses, with main terms for T2 location (different object, different diagonal) and T1-T2 lag, revealed a significant main effect of lag $[F(4,36)=8.22, p<.001]$, due to an increase in T2 accuracy with increasing T1-T2 lag (from $54 \%$ to $76 \%$ for lags from 130 to $390 \mathrm{msec}$ ). The main effect of T2 location was nonsignificant $[F(1,9)=3.73$, $p=.08]$; if anything, the AB was slightly larger, by $3.6 \%$, for the (farther) diagonally opposite than for the (nearer) different object T2 location (see Figure 3C).

\section{Discussion}

Experiment 2 clearly replicated the results obtained in Experiment 1, in showing robust object-based costs of $\sim 14 \%$ at short temporal lags (i.e., below $400 \mathrm{msec}$ ). Moreover, T2 identification performances for the two RSVP stream locations within the same object were nearly identical. This pattern of results shows that the AB is not simply determined by the spatial distance between the $\mathrm{T} 1$ and T2 locations, but by whether or not T2 is located within the same or a different global object, relative to T1: Besides this object-based effect, there was no further, significant spatial distance effect. (Although this appears to be at variance with Kristjánsson and Nakayama [2002], it may simply be that the range of spatial distances examined in Experiment 2 was too small to demonstrate such effects. Also, the global [bar] objects might have obscured potential spatial distance effects.)

Note that in the same-position condition, the absence of a location (and a task) switch should, in principle, have resulted in lag 1 sparing (e.g., Juola et al., 2004; Visser, Bischof, \& Di Lollo, 1999). However, the analysis of the same-position data (Figure 3B) failed to show spared lag 1 performance (if anything, there was a nonsignificant increase of $\sim 6 \%$ for the same-position condition, relative to the same-object condition). This outcome appears, at 
A

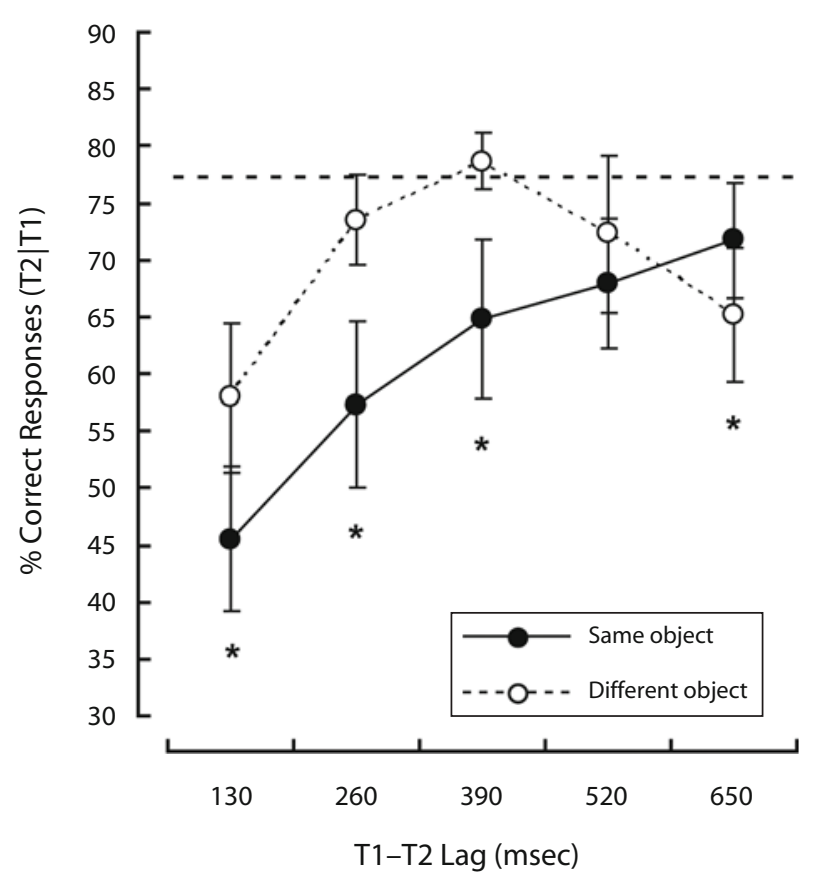

B

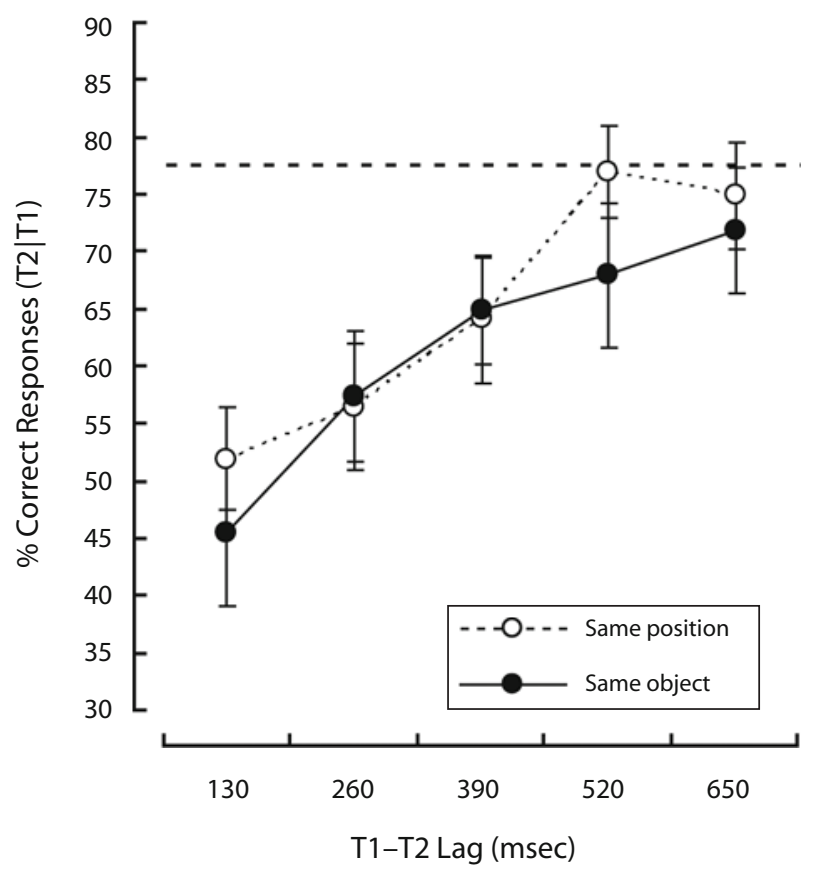

C

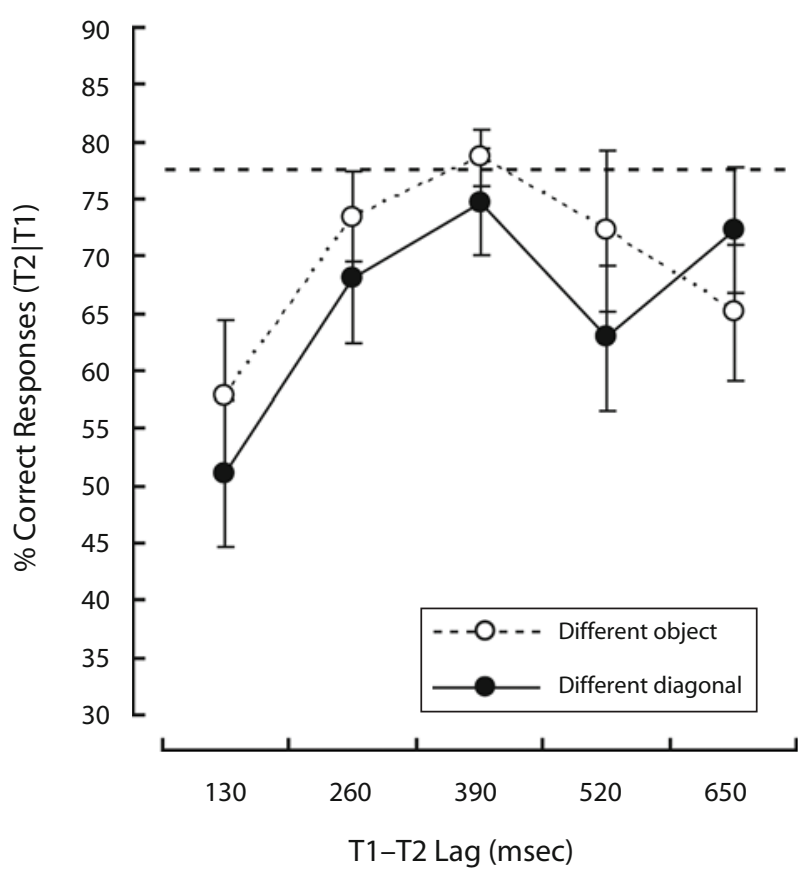

Figure 3. Mean percentages of correct identifications of the second target (T2), given correct identifications of the first target (T1) in Experiment 2. Correct identifications are presented as a function of the temporal lag between T1 and T2. (A) Comparison of same-object (solid line) and different-object (dashed line) conditions. (B) Comparison of T2 positions presented within the same bar as T1. (C) Comparison of T2 positions that were presented on a different bar from T1. Significant differences between conditions are indicated by asterisks. The dashed horizontal lines indicate the level of overall T1 accuracy. Error bars indicate the standard errors of the means. 
first, inconsistent with the existing literature. However, a possible explanation may be derived from Olivers and Meeter (2008), who showed that lag 1 sparing is not simply dependent on the sequence of two target items but, crucially, on their temporal separation, with reliable sparing being evident only with lags shorter than $100 \mathrm{msec}$ (see also Potter, Staub, \& O'Connor, 2002). On this basis, one would not expect lag 1 sparing at the shortest SOA in the same-position condition in Experiment 2, because the T1-T2 lag was $130 \mathrm{msec}$ (i.e., >100 msec).

In summary, the outcome of Experiment 2 confirms an object-based modulation of the $\mathrm{AB}$ in terms of an increased cost for any $\mathrm{T} 2$ presented within the same object as T1 (as compared with any T2 presented within a different object, relative to T2), while showing that the unequal distribution of T2 locations in Experiment 1 did not affect the overall pattern of results.

\section{EXPERIMENT 3}

The two experiments presented thus far show that, at short temporal lags $(<400 \mathrm{msec})$, the $\mathrm{AB}$ is stronger when two targets are presented within the same object, rather than on different objects. In Experiment 3, we set out to explore in further detail the type of object representation that underlies this effect. One potential explanation for object-based costs could be that attentional effects (whether facilitatory or inhibitory) spread less effectively across luminance borders than within regions of the same brightness. On the other hand, however, object-based attention has been shown to operate on relatively complex object representations that require perceptual completion (e.g., Haimson \& Behrmann, 2001; Moore \& Fulton, 2005; Moore, Yantis, \& Vaughan, 1998). In particular, these studies demonstrate that object-based attention can spread even across hidden portions of a partially occluded object (see Figure 4 for an example configuration), suggesting that attentional processes operate on completeobject representations.

To examine the object representation underlying the $\mathrm{AB}$ modulation demonstrated in the previous experiments, in Experiment 3, we investigated the role of perceptual completion in the object-based modulation of the AB. Displays consisted of two vertical bars, with the four RSVP streams presented, one at each end of a bar. In addition, a central horizontal bar was added that occluded the two vertical bars (see Figure 4 for an example display). Thus, if the AB modulation is confined to regions within luminance borders, no object-based cost should be observable in Experiment 3. Alternatively, if the modulation is based on complete-object representations, the occluded objects should show a sameobject cost similar to that in the previous experiments.

\section{Method}

Experiment 3 was identical to Experiment 1, except for the configuration of the global (bar) objects. On each trial, two black, vertical outline rectangle bars were presented together with a third, horizontal bar (subtending $2.3^{\circ} \times 11.6^{\circ}$ ) displayed at the center of the screen so that it occluded the two vertical bars. The thickness of the black bar outlines was 2 pixels. The screen background and the sur-

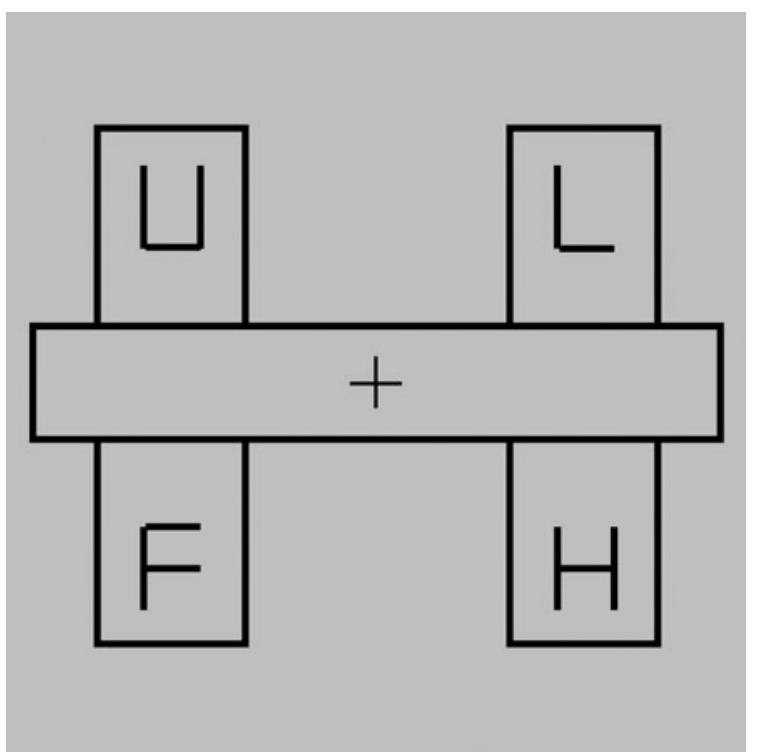

Figure 4. Example of the occluded displays employed in Experiment 3.

faces of the bars were all presented in gray (luminance: $3.81 \mathrm{~cd} / \mathrm{m}^{2}$ ). As in the previous experiments, four RSVP streams were presented at each of the four vertical bar ends. Figure 4 presents an example of a display configuration. All other details were identical to those in Experiment 1. Ten paid observers ( 4 of them male; mean age $=$ 28.2 years; normal or corrected-to-normal visual acuity) participated in the experiment. In summary, the experiment varied the factors of T2 location (same object, different object), and T1-T2 lag (130, 260, 390,520 , or $650 \mathrm{msec}$ ), with all possible factorial combinations presented in random order. Each observer completed 40 practice plus 400 experimental trials (divided into 10 blocks).

\section{Results}

T1 identification was again found to be quite accurate, with an average of $74 \%$ of correct responses, comparable to the level of performance in the previous experiments.

Figure 5 presents the accuracy of the $\mathrm{T} 2$ responses (given a correct T1 response) as a function of lag, separately for the same- and different-object conditions. A two-way repeated measures ANOVA of correct T2 responses, with main terms for T2 location (same object, different object) and T1-T2 lag $(130,260,390,520,650 \mathrm{msec})$, revealed both main effects to be significant: T2 location $[F(1,9)=$ $17.03, p<.003]$ and $\operatorname{lag}[F(4,36)=26.71, p<.001]$. Accuracy was higher when T2 occurred within a different- as compared with a same-object T2 location (66\% vs. $59 \%)$. In addition, accuracy increased with lag $(46 \%, 56 \%, 64 \%$, $70 \%$, and $76 \%$ for lags of $130,260,390,520$, and $650 \mathrm{msec}$, respectively). The interaction was not significant ( $p=$ .27 ), even though same-object costs were obtained more reliably for the lags from 130 to $520 \mathrm{msec}$ than for the 650-msec lag [same-object costs (and associated $p$ values) were $12 \%(p<.02), 7 \%(p=.1), 10 \%(p<.03), 5 \%$ $(p=.06)$, and $-1 \%(p=.39)$ for lags of 130, 260, 390, 520 , and $650 \mathrm{msec}$, respectively]. Thus, same-object costs of $\sim 9 \%$ were obtained for lags of up to $500 \mathrm{msec}$, in line with the findings of Experiments 1 and 2. 


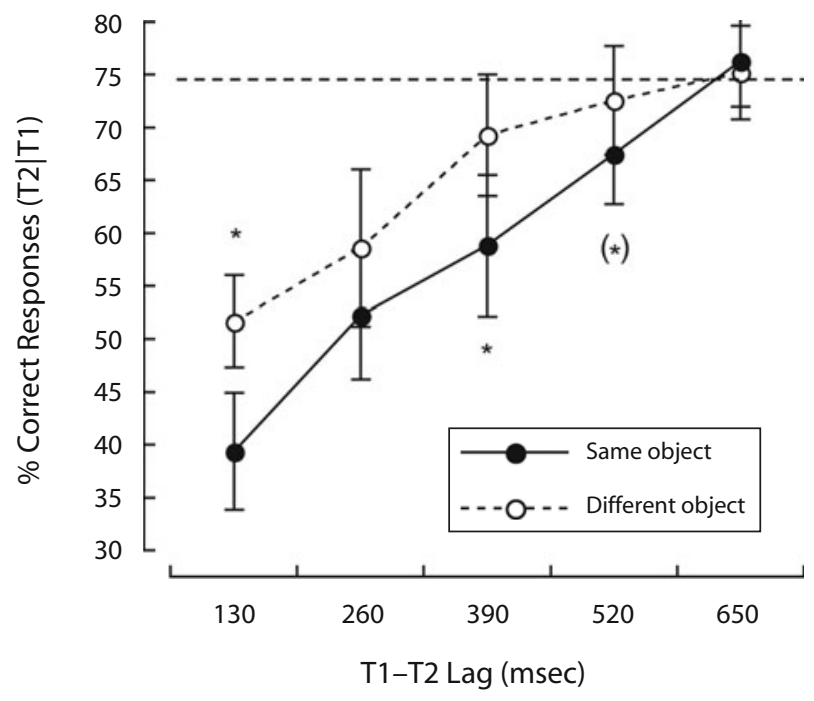

Figure 5. Mean percentages of correct second-target (T2) identifications, given correct identifications of the first target (T1) in Experiment 3 (presenting occluded vertical bars). Correct identifications are presented as a function of the temporal lag from the onset of T1 to the onset of T2, separately for same-object (solid line) and different-object (dashed line) conditions, with significant differences between the same- and different-object conditions indicated by asterisks, and a marginally significant difference by an asterisk within parentheses. In addition, the dashed horizontal line indicates the level of overall T1 accuracy. Error bars indicate the standard errors of the means.

\section{Discussion}

In Experiment 3, we examined whether object-based costs in the $\mathrm{AB}$ would generalize to configurations that presented hidden portions of an occluded object. The results indicated that this was indeed the case. Reliable object-based costs of $\sim 9 \%$ were obtained for the lags from 130 to $520 \mathrm{msec}$. This pattern of results is consistent with that of previous studies, while additionally showing that object-based modulations survive occlusion not only in facilitatory (spatial) object cuing (Haimson \& Behrmann, 2001; Moore \& Fulton, 2005; Moore et al., 1998), but also in the inhibitory tagging of locations within an attended object in the $\mathrm{AB}$ paradigm.

\section{EXPERIMENT 4}

In Experiment 4, we set out to investigate an apparent inconsistency between the present results and previous findings that have also revealed an object-based influence on the AB. As was demonstrated in Experiments 1-3, at short temporal lags, identification of T2 is more impaired if it is presented within the same, as compared with a different, object. By contrast, Raymond (2003; see also Kellie \& Shapiro, 2004) reported that identification of T2 is less impaired if it involves the same basic object as T1 (i.e., an additional line segment added, or uniformly connected, to a particular prototype object, either a trident or an arrowhead). However, the two paradigms were dissimilar in many, potentially critical aspects, in particular, with respect to (1) the type of object (global [responseirrelevant] grouping in the present experiments vs. local, uniformly connected line segments [response-relevant] in Raymond, 2003) and (2) the type of target (whole letters presented within the global groupings vs. line components of local object structures). Despite these differences, both paradigms are coherent in that they demonstrate an influence of object information on the AB. Nevertheless, the results are conflicting in terms of the direction of effects (costs vs. benefits).

Consequently, to directly compare both approaches, Experiment 4 was designed to combine the different aspects characterizing the two paradigms within a single experiment: Although the global object layout was identical to that in Experiment 2, in Experiment 4, the local targets could be only one of two letters $(\mathrm{S}$ or $\mathrm{H})$ that were either repeated (i.e., T1 and T2 were the same) or not (i.e., T1 and $\mathrm{T} 2$ were different). This was meant to be similar to the local-object variation in Raymond (2003), in that identical or nonidentical (response-relevant) letters are instances of either a same- or a different-object prototype.

\section{Method}

Experiment 4 was basically identical to Experiment 2 (including presentation of $\mathrm{T} 2$ at the same and diagonally opposite positions, relative to T1), except for the possible identities of the targets and nontargets. The targets were an uppercase $\mathrm{S}$ or $\mathrm{H}$, and the nontargets could be six different uppercase letters (U, E, P, C, F, L). Nontargets were presented in a randomized sequence of 16 (black) letters, with each of the 6 possible letters presented for up to three times within a given stream. The two (red) targets, T1 and T2, were either repeated (same target: SS or $\mathrm{HH}$ ) or not (different target: $\mathrm{SH}$ or HS). In addition, the SOA was reduced to $110 \mathrm{msec}$, in order to slightly increase task difficulty (a pilot test showed that observers were close to perfect response accuracy with 130-msec SOAs). All other details were identical to those in Experiment 2. Ten paid observers ( 5 of them male; mean age $=26.0$ years; normal or corrected-to-normal visual acuity) participated in the experiment. In summary, Experiment 4 varied the factors of $T 2$ location (same position, same object, different object, or different diagonal), local target (same target, different target), and T1-T2 $\operatorname{lag}(110,220,330,440$, or $660 \mathrm{msec})$, with all possible factorial combinations presented in random order. Each observer completed two sessions, each of 40 practice plus 400 experimental trials (divided into 10 blocks).

\section{Results}

Accuracy of T1 identifications was very high, with an average of $95 \%$ correct identifications. That is, despite the shorter SOA, more correct responses were given in Experiment 4 than in the previous experiments [e.g., when T1 performance is compared for Experiments 2 and 4, $77 \%$ vs. $95 \%$, respectively; $t(18)=4.51, p<.001]$.

Global objects. For the analysis of T2 responses (given a correct $\mathrm{T} 1$ response), in a first step, the sameand different- (global) object conditions were compared (i.e., conditions with equivalent spatial T1-T2 distances, as in the previous experiments). A three-way repeated measures ANOVA of correct T2 responses, with main terms for global object (same object, different object), local target (same target, different target), and T1-T2 lag $(110,220,330,440,660 \mathrm{msec})$, revealed significant main 
effects for global object $[F(1,9)=12.22, p<.008]$, local target $[F(1,9)=5.18, p<.05]$, and $\operatorname{lag}[F(4,36)=12.77$, $p<.001]$. For the global objects, accuracy was higher for different- than for same-object T2 locations ( $94 \%$ vs. $90 \%$ ). Similarly, for local targets, accuracy was higher for the different-target condition than for the same-target condition ( $94 \%$ vs. $90 \%$ ). In addition, performance increased from lag 1 to lag $3(86 \%, 90 \%, 94 \%, 95 \%$, and $96 \%$ for lags of 110, 220, 330, 440, and $660 \mathrm{msec}$, respectively).

Moreover, there were significant interactions. First, the interaction of global object and lag $[F(4,36)=3.71, p<$ .02 ] was significant. Figure $6 \mathrm{~A}$ presents T2 accuracy as a function of lag, separately for the same- and differentobject conditions. As can be seen, significant same-object costs of $\sim 7 \%$ were obtained for lags of $<300 \mathrm{msec}$ (all $p$ s $<.05)$, whereas no significant differences were observable at longer lags. Second, the interaction of local target and lag $[F(4,36)=3.77, p<.02]$ was significant. Figure $6 \mathrm{~B}$ presents $\mathrm{T} 2$ accuracy as a function of lag, separately for the same- and different-target conditions. As for the global objects, the local targets showed a cost of $\sim 8 \%$ at lags of $<300 \mathrm{msec}$ for same, as compared with different, targets (all $p \mathrm{~s}<.05$ ); this effect vanished at longer lags. Thus, this pattern of results shows that both globaland local-object configurations engender comparable same-object costs at short temporal lags, comparable to the results presented for Experiments 1-3. Note, however, that for this analysis, T2 always occurred at a neighboring (i.e., distance-controlled) position, relative to $\mathrm{T} 1$.

Local targets. A second analysis was performed to examine whether the effect of local-target repetition (impaired performance for identity repetitions vs. changes) is influenced by T2 location (same position as T1, same object, different object, or different diagonal). Figure 7 presents T2 accuracy as a function of lag for identical and nonidentical targets, separately for the same-position (7A), same-object (7C), different-object (7B), and differentdiagonal (7D) conditions. A repeated measures ANOVA of correct T2 responses (given a correct T1 response), with main terms for T2 location, local target (same target, different target), and T1-T2 lag $(110,220,330,440$, $660 \mathrm{msec})$, revealed a main effect for $\operatorname{lag}[F(4,36)=23.35$, $p<.001$ ], reflecting an increase in performance from lag 1 to lag $3(85 \%, 90 \%, 93 \%, 93 \%$, and $94 \%$ for lags of 110 , 220, 330, 440, and $660 \mathrm{msec}$, respectively). Moreover, the interaction of $\mathrm{T} 2$ location and local target $[F(3,27)=$ $3.51, p<.03]$ and the three-way interaction $[F(12,108)=$ $5.18, p<.001]$ were significant. These interactions were due to the same-target condition's showing an advantage of $7 \%$ (relative to the different-target condition) when the spatial location remained the same; by contrast, when the position switched from $\mathrm{T} 1$ to $\mathrm{T} 2$, the same-target condition was less accurate than the different-target condition (costs were $3 \%, 3 \%$, and $4 \%$ for the same-object, differentobject, and different-diagonal conditions, respectively). Furthermore, these differences between targets at same and different spatial positions were especially pronounced at the lag of $110 \mathrm{msec}$ (significant three-way interaction). As can be seen in Figure 7A, for the same positions, the different-target condition showed a large drop in accuracy (by $22 \%, p<.05$ ) at the 110 -msec lag, whereas there was no pronounced $\mathrm{AB}$ effect for the same-target condition. By contrast, the reverse - namely, a drop in accuracy for the same-target condition (by $11 \%$; all $p \mathrm{~s}<.05$ ) - occurred at the 110-msec lag for all position changes (see Figures 7B7D). Whereas the pattern of effects for same positions of 1
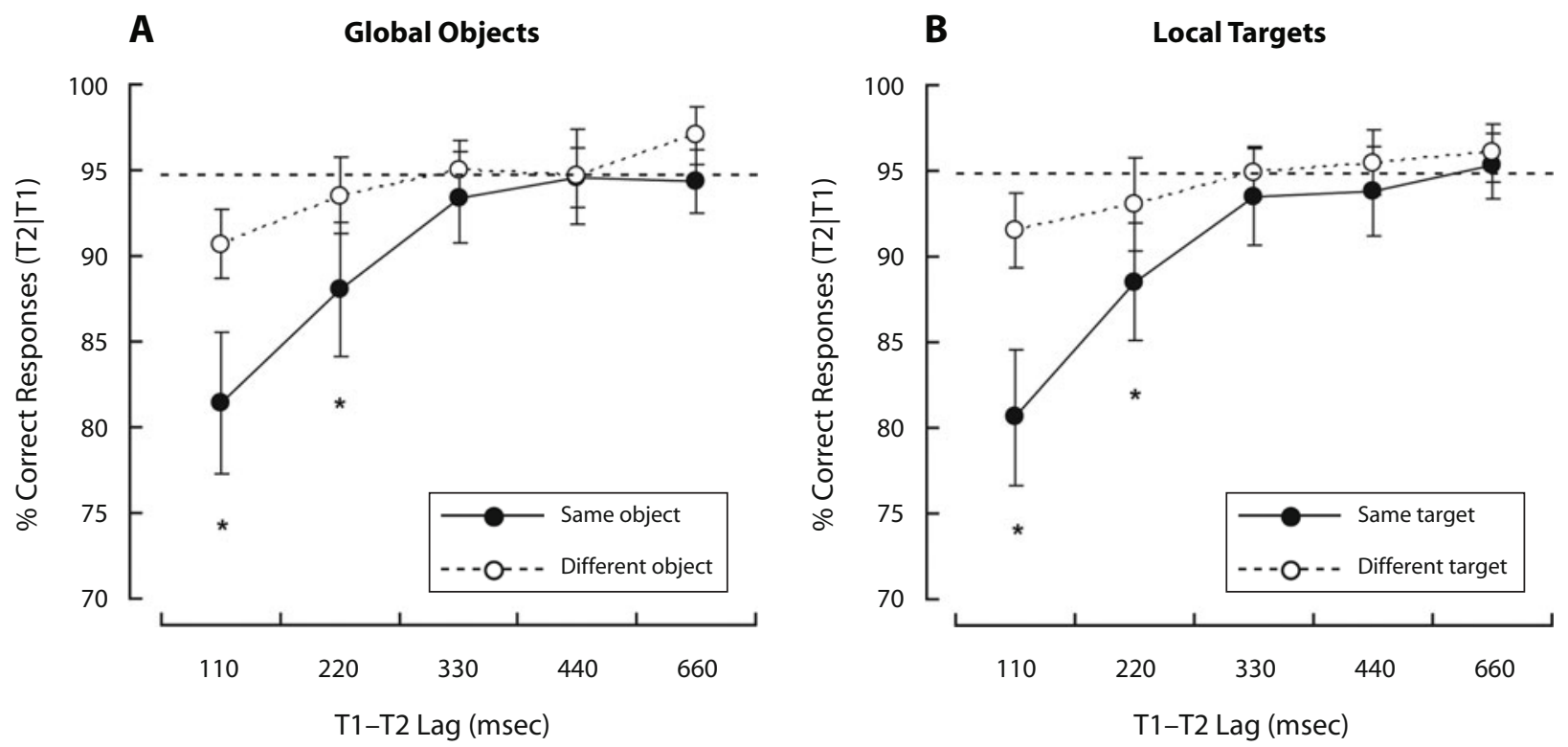

Figure 6. Results from Experiment 4: (A) Mean percentages of correct T2 identifications, given a correct T1 response for the sameobject versus different-object comparisons at equal spatial distances. (B) Differences between target repetitions (same target) and target switches (different targets), again for controlled spatial distances. Significant differences between conditions are indicated by asterisks. The dashed horizontal lines indicate the level of overall T1 accuracy. Error bars indicate the standard errors of the means. 


\section{A}

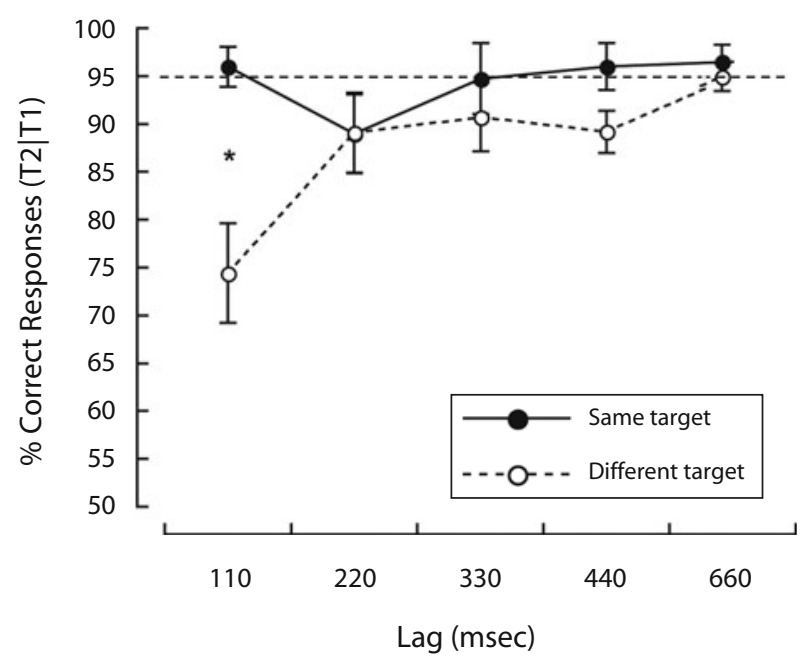

C

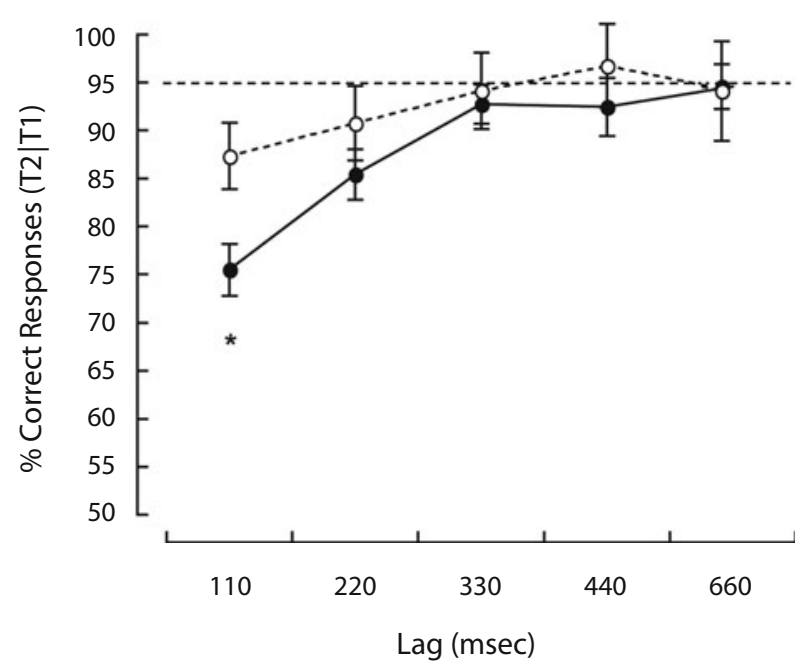

B Different Object

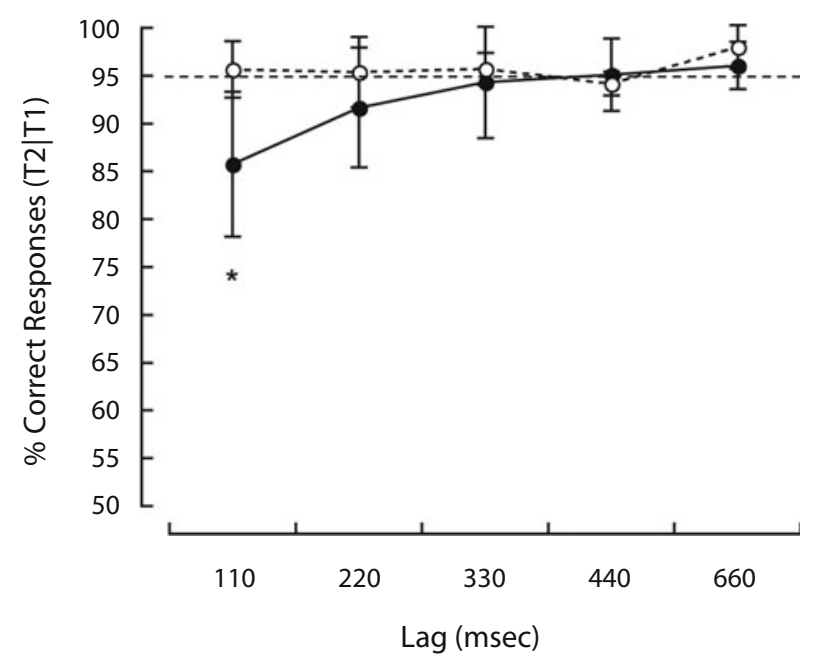

D Different Diagonal

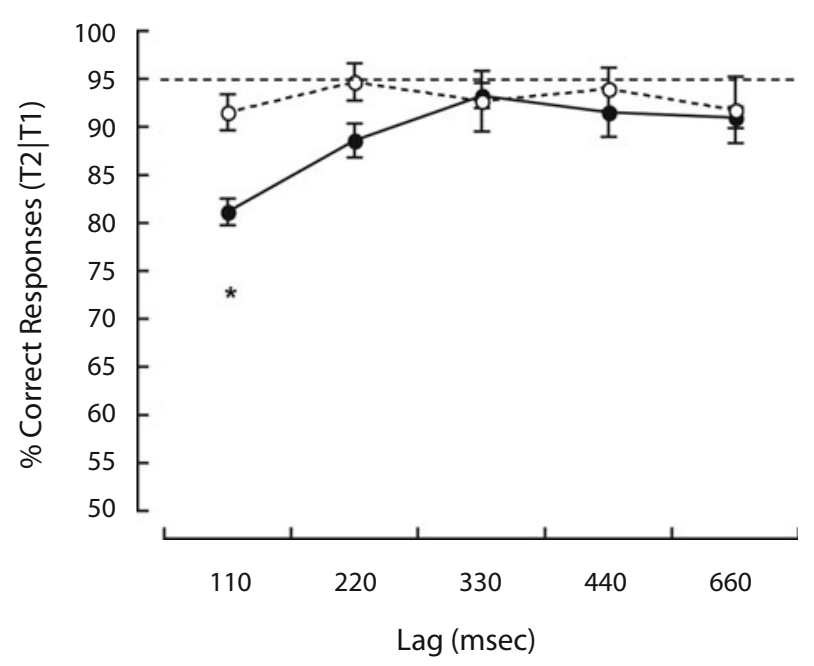

Figure 7. Comparisons of target repetitions and switches in Experiment 4: Each panel depicts the mean percentages of correct T2 identifications, given a correct $\mathrm{T} 1$ response for the same-position condition (A), the different-object condition (B), the same-object condition (C), and the different (object) diagonal condition (D). Significant differences between pairwise comparisons are indicated by asterisks. The dashed horizontal lines indicate the level of overall T1 accuracy. Error bars indicate the standard errors of the means.

and $\mathrm{T} 2$ corresponds to the same-object benefits described by Raymond (2003), a position switch caused same-object costs comparable to the present results.

\section{Discussion}

Experiment 4 was meant to combine global- and localobject-based effects within a single experiment. The results showed effects for both types of grouping (despite an overall increased level of accuracy due to changes in task demands). On the one hand, repetitions of the global (bar) objects showed results comparable to those in our previous experiments - namely, a pronounced same-object cost for short temporal lags $(<300 \mathrm{msec})$. On the other hand, repetitions of the local (target) objects showed a pattern of effects that was dependent on the spatial position: When the target position was the same for $\mathrm{T} 1$ and $\mathrm{T} 2$, no $\mathrm{AB}$ was observable for identically repeated targets, whereas changes in target identity (from T1 to T2) caused a pronounced drop in performance at the $110-\mathrm{msec}$ lag. This outcome replicates the findings of Raymond (2003) and Kellie and Shapiro (2004). However, when the target position changed from $\mathrm{T} 1$ to $\mathrm{T} 2$, the reverse pattern of effects was observed - namely, a strong AB at the 110-msec lag for repetitions of target identity, but no pronounced decrement in performance for identity changes. Thus, the same-object benefit reported in previous studies (e.g., Raymond, 2003) is confined to single spatial locations, whereas a same-object cost can occur for both shifts of attention within a global object and when the local (uniformly connected) object shifts its position. 


\section{GENERAL DISCUSSION}

The objective of the present experiments was to investigate whether global-object information would have an influence on the $\mathrm{AB}$. The results of four experiments revealed this to be the case. Experiment 1 showed an object-based modulation of performance. Significant same-object costs were found when the second target followed the first within $400 \mathrm{msec}$ (irrespective of whether $\mathrm{T} 1$ identification was correct or incorrect): When T2 was presented on the same bar as $\mathrm{T} 1$, the $\mathrm{AB}$ was more marked than when T2 was presented on a different bar (at identical spatial distances). A similar pattern of results was obtained in Experiment 2, in which T2 could occur at any of the four possible locations: When T2 was presented in one of the two RSVP streams within the same bar as T1, performance for lags up to $400 \mathrm{msec}$ was more impaired than when $\mathrm{T} 2$ was presented in a stream within a different bar. Furthermore, Experiment 3 showed that object-based $\mathrm{AB}$ effects can also occur for occluded objects: Reliable same-object costs were evident even though the two bars that contained the RSVP streams were partially hidden behind an occluder. This indicates that the object-based modulations of the $\mathrm{AB}$ demonstrated in the present experiments are integrated on the basis of mechanisms that subserve perceptual completion (e.g., Moore et al., 1998). Finally, in Experiment 4, object-based effects were compared at both global and local levels of representation (i.e., for the global bars and for the local-target identities). Whereas for the global (bar) objects, same-object costs essentially replicated the results in Experiments 1-3, repetition of the local-target identities engendered either costs or benefits, depending on whether or not T1 and T2 were spatially separated. Specifically, T2 identification was largely unaffected when both target identities and locations were repeated or when both target identities and locations changed. By contrast, an $\mathrm{AB}$ (with a specific drop in performance at lag 1 ) was found when either only the T2 identity or only the T2 location was changed. This finding shows that the integration of location and identity information is crucial in determining whether objectbased effects turn into benefits or costs.

All in all, the present demonstration of an object-based modulation of the $\mathrm{AB}$ is in line with the view that the allocation of attention is influenced by grouped (object) arrays (Driver \& Baylis, 1989; Duncan, 1984; Egly et al., 1994; Lamy \& Egeth, 2002; O'Grady \& Müller, 2000; Vecera \& Farah, 1994; Watson \& Kramer, 1999). Thus, object-based effects in RSVP task performance not only may relate to single, uniformly connected objects at a fixed spatial location (Kellie \& Shapiro, 2004; Raymond, 2003), but also may arise from global-object groupings that extend across separate positions in space. However, importantly, both types of (global or local) objects differ substantially in the direction of their effects.

\section{Global Objects}

All four experiments revealed that global-object (bar) groupings led to a temporary ( $400 \mathrm{msec})$ increase in the
$\mathrm{AB}$, so that $\mathrm{T} 2$ identification was always found to be more impaired if it was located at a neighboring position within the same object as T1, as compared with an equidistant position within a different object. This suggests that the $\mathrm{AB}$ spreads preferentially across the entire (global) object.

Note that such a finding of same-object costs appears to be at variance with studies of object-based attention in the spatial domain, which have typically yielded sameobject advantages - that is, expedited selection of a target that occurred within the same global object as a preceding cue (e.g., Egly et al., 1994; Lamy \& Egeth, 2002; Watson $\&$ Kramer, 1999). Yet, assuming that T1 acts as a spatialobject cue, the present results revealed a same-object cost for the subsequently presented T2. How can this reversal be explained?

One potential explanation could be in terms of objectbased inhibition of return (IOR; e.g., Tipper, Driver, \& Weaver, 1991). On this account, same-object costs would arise because inhibitory tags prevent the visual system from reinspecting already attended objects. However, object-specific inhibition should be observable in standard spatial-cuing paradigms, as well as in the present multipleRSVP-stream paradigm. However, no IOR-like pattern (of same-object costs) was evident in either of the relevant studies (Egly et al., 1994; Lamy \& Egeth, 2002); rather, these reported same-object benefits at cue-target SOAs comparable to the $\mathrm{T} 1-\mathrm{T} 2$ lags in the present experiment (100- to 300-msec SOAs in spatial-cuing experiments, as compared with 130 - to 390 -msec lags in the present study). Moreover, an IOR pattern in an Egly et al.-type spatialcuing experiment has been observed only much later, after an SOA of 1,000 msec (Jordan \& Tipper, 1999; List \& Robertson, 2007). In general agreement with these late IOR effects, a study that combined spatial cuing with a two-stream RSVP paradigm revealed an IOR pattern only after $\sim 700 \mathrm{msec}$ (following an early facilitatory effect; see Klein \& Dick, 2002). Thus, these temporal characteristics would argue against an IOR-based account, given that the present object-based $\mathrm{AB}$ effects occurred mostly before $\sim 400 \mathrm{msec}$ (whereas there was no indication of an IOR for the late, 650-msec lag).

Nevertheless, IOR may critically depend on the need to actively encode the first stimulus prior to encoding the second one. Although this is a requirement in the multiRSVP-stream paradigm, it is not in the standard spatialcuing paradigm, in which the cue is response irrelevant. However, Lamy and Egeth (2002) showed reliable objectbased effects (i.e., different-object costs) to be manifest in a task that required same-different judgments of asynchronous targets presented at separate positions, as well as in standard spatial-cuing tasks. Thus, it would appear that the present finding of a same-object cost is specific to the multi-RSVP-stream paradigm and is not explicable in terms of more general mechanisms of object-based IOR.

Given that an object-based IOR account is not feasible, another alternative would be that same-object costs in the multi-RSVP-stream paradigm arise at a higher level, one at which visual stimulus representations are encoded and consolidated into temporary object files (Kahneman \& Treis- 
A

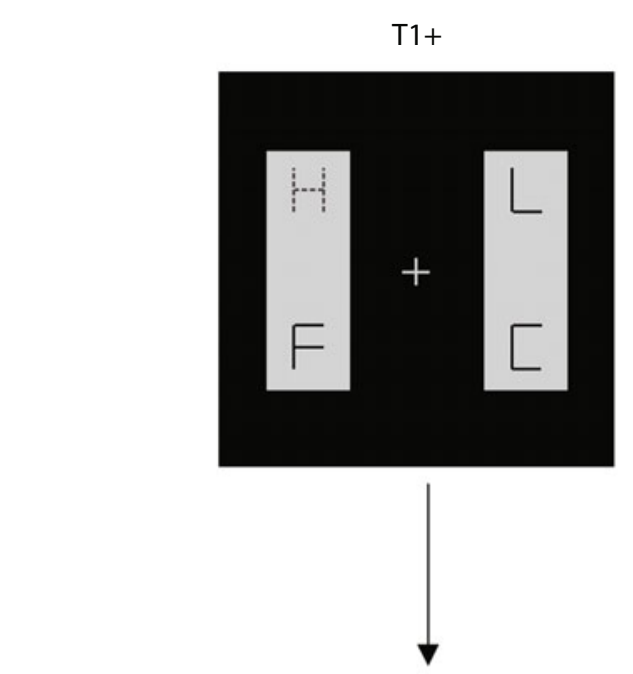

T2-

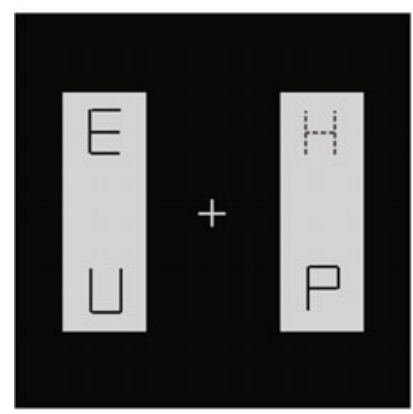

$\mathrm{T} 2+$

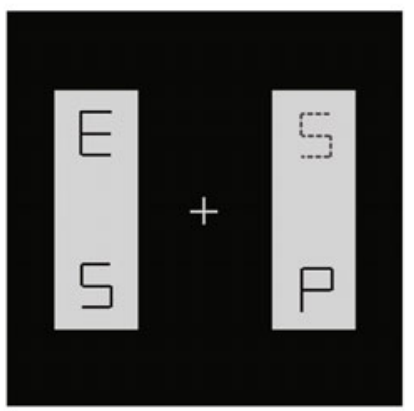

B

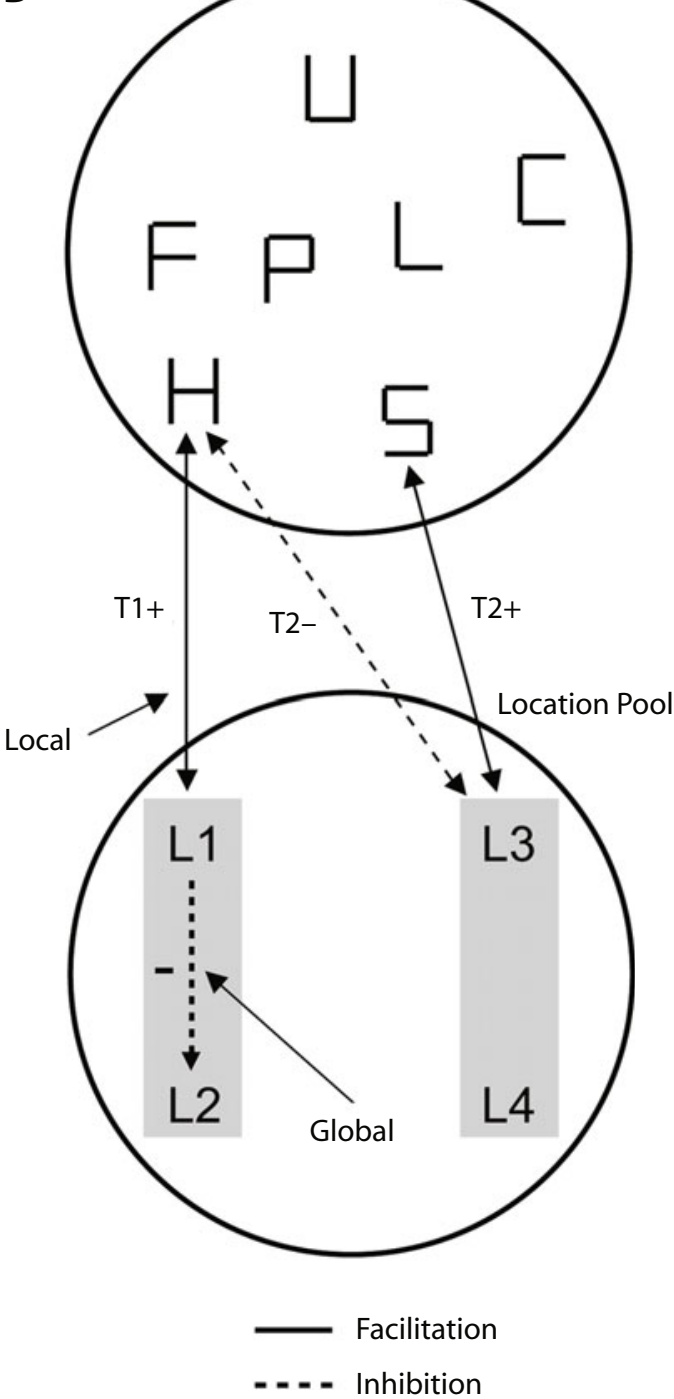

Figure 8. Example T1-T2 sequences (A) and schematic model (B) to account for local- and global-object-based attentional blink effects. In the example sequence (A), the first target, $T 1$ (the dashed letter $\mathrm{H}$ ), initializes an object file that consists of a facilitatory connection $(\mathrm{T} 1+)$ between the identity of the target (represented in a pool of nodes for each possible target identity) and its location (represented in a pool of nodes for each possible target location, L1-L4). If the subsequent T2 appears at a new position (L3) but shares the identity of T1, interference arises (T2-) because the required identity node $(\mathrm{H})$ is already bound to a different location $(\mathrm{L1})$. However, if $\mathrm{T} 2$ differs from $\mathrm{T} 1$ in both identity and location (e.g., an $\mathrm{S}$ at $\mathrm{L3}$ ), a second facilitatory connection can be readily established (T2+) between nonoccupied identity and location nodes. In addition, besides the local connections linking target identity and location nodes, an inhibitory influence is triggered by $\mathrm{T} 1$ between locations grouped within the same global (vertical/horizontal bar) structure (in the example, vertical inhibitory connection from L1 to L2). This realizes an inhibitory linkage of local-object files via global grouping of object locations. See the text for further details.

man, 1984) for later report. According to this account, after T1 identity is registered in the file of the cued object, this file may be "locked" for updating for some time, interfering with the registration, within the same (or a related) file, of a same-object T2. Crucially, this account assumes that there is some sort of object-based file linkage between sameobject (but not different-object) T1 and T2 stimuli and that (linked) file updating exhibits a refractoriness - akin to a form of conceptual, as opposed to spatial, inhibition. Indeed, Houghton and Tipper (1994) have argued that IOR not only is expressed in spatial terms, but may also involve inhibition in conceptual-object memory-terms (which may, in turn, enhance spatial inhibitory processes in a topdown manner). Accordingly, conceptual object-based inhibition, in terms of the inhibited updating of linked-object files, would provide the most coherent explanation for the present pattern of effects. In terms of the level of processing concerned, this proposal would be consistent with other accounts that have attributed the AB to limited-capacity mechanisms operating at conceptual, object-related levels of representation (Chun \& Potter, 1995; Kellie \& Shapiro, 2004; Raymond, 2003). 


\section{Single Uniformly Connected Objects}

A somewhat different outcome was obtained for localobject prototypes: In agreement with Raymond (2003) and Kellie and Shapiro (2004), Experiment 4 showed that, for single, uniformly connected objects, item (and location) repetitions reduce the $\mathrm{AB}$. Raymond suggested that this same-object benefit can be ascribed to a process whereby a visual stimulus representation is encoded and consolidated into a temporary object file. Within this framework, an $\mathrm{AB}$ reduction (for uniformly connected objects) would occur when an object file has already been created (in order to represent T1), so that, when a same-object T2 arrives, the identical object file needs only to be updated. By contrast, when T2 constitutes a different-object representation, a new object file must be opened, and this additional process leads to an $\mathrm{AB}$.

However, as was demonstrated in Experiment 4, the absence of an $\mathrm{AB}$ with single, uniformly connected objects was obtained only when both the location and identity of the two targets repeated or when both the identity and location switched. By contrast, an increased $\mathrm{AB}$ was found when either only the identity or only the location of $\mathrm{T} 2$ was changed. ${ }^{1}$ This suggests that the efficient updating of an existing object file is specifically linked to its spatial position. Thus, a given object file would store not only the identity, but also the location of a given target (even though the location information is not response relevant).

A plausible account for this pattern of results may be provided by the schematic model illustrated in Figure 8B, along with an example T1-T2 sequence on a given trial (Figure 8A). The model assumes that object files consist of linkages (bindings) between activated nodes in separate pools, one for target identity (features) and one for location, and that these linkages are mutually exclusive, so that only one identity node can be bound to one location node and vice versa (more precisely, there is a bias against binding the same identity node to multiple location nodes, and vice versa). Accordingly, in the example T1-T2 sequence (Figure 8A), the first target, the letter $\mathrm{H}$, would be registered in an object file that consists of an excitatory connection between the target's identity, $\mathrm{H}$, and its spatial location, L1 (see link T1 + in Figure 8B). If the subsequent target, T2, has the same identity and is presented at the same location as T1, no interference would arise, since the already established and open object file would only require updating (i.e., registering that it encodes T2 as well as T1). Furthermore, in contrast to previous notions (e.g., Raymond, 2003), the creation of a second object file would not per se lead to an AB. In particular, a T2 that is different in identity (e.g., the letter S) and appearing at a different location (e.g., L3) would not give rise to interference (see link $\mathrm{T} 2+$ in Figure 8B), because the to-be-created T2 object file has no nodes in common with the already existing T1 file. However, an increased AB would emerge in cases in which $\mathrm{T} 1$ and $\mathrm{T} 2$ are partially overlapping, in either identity or location (e.g., when T2 is also an $H$ but is presented at a different location, L3), because of a bias against linking the same identity node (which is already bound by T1) to a different location node, or vice versa (as when a different identity node would need to be linked to the same location). As a result, any partial overlap, in identities or locations, between T2 and T1 would inhibit the creation of a new object file (see T2 - in Figure 8B), leading to a transient drop in T2 identification performance (as was observed in Experiment 4). Thus, within this framework, the magnitude of the $\mathrm{AB}$ results from the degree to which object files - that is, linkages between target identity and location nodes - can be established without overlap.

This schematic model can also capture the global-object $\mathrm{AB}$ effects (i.e., the especially reduced performance for a T2 within the same outline rectangle as T1) observed in Experiments 1-4: Global-object-based costs would be explicable if it is additionally assumed that the files for local objects within a global spatial structure, such as the rectangular bar, are subject to extra inhibition that spreads across locations within the global grouping. Thus, if T1 is selected at one location (L1) within a given global object, linked locations within the same global structure are especially inhibited (see the inhibitory connection from L1 to L2 in Figure 8B), making it harder to include the corresponding location node (L2) in the to-be-created T2 object file. In effect, this would realize a (inhibitory) linkage of local object files via the global grouping of object locations. The latter would explain why the $\mathrm{AB}$ is generally larger for a $\mathrm{T} 2$ within the same spatial structure as $\mathrm{T} 1$ and, specifically, why there is an $\mathrm{AB}$ even when $\mathrm{T} 2$ is different in identity to T1. Recall that, if T2 is not part of the same global structure as $\mathrm{T} 1$, there is no $\mathrm{AB}$ for an identity change (compare Figures 7B vs. 7C).

The assumption of a spatial interaction between separate RSVP stream locations is also consistent with Kristjánsson and Nakayama's (2002) proposal that attentional resolution is sensitive to the spatial and temporal separation between two AB targets. Specifically, Kristjánsson and Nakayama proposed the idea of a spatiotemporal gradient of attentional resolution, on the basis of their finding that the spatial (and temporal) distance between two sequential targets determines the magnitude of the $\mathrm{AB}$. Such a spatiotemporal gradient could be integrated within a location-specific representation of sequential targets (as, for example, in Figure 8B). In addition, global-object groupings could be represented within the location pool, determining how attention is (re)allocated. Thus, if the spatial distribution of potential target positions is assumed to be biased by global processes of region segmentation (e.g., Conci, Müller, \& Elliott, 2007), the AB is not simply determined by a spatiotemporal gradient, but also by global object boundaries (resulting in an inhibitory spread of activity within corresponding locations). Thus, selection of a T2 will be more impaired (within a critical time period) if a T1 had previously been selected within the same global object (as opposed to a different global object), due to the spreading of inhibitory activity across the global object.

\section{AUTHOR NOTE}

This work was supported by Deutsche Forschungsgemeinschaft (DFG) Research Group (FOR 480) and CoTeSys Excellence Cluster (142) grants. We thank Barbara Neumayer, Sonja Riegert, and Philipp Schiebler for help with running the experiments, and Arni Kristjánsson, 
Dominique Lamy, Jeremy Wolfe, and an anonymous reviewer for valuable comments on an earlier draft of the manuscript. Correspondence concerning this article should be addressed to M. Conci, Allgemeine und Experimentelle Psychologie, Department Psychologie, LudwigMaximilians Universität, Leopoldstr. 13, D-80802 Munich, Germany (e-mail: conci@psy.uni-muenchen.de).

\section{REFERENCES}

Brainard, D. H. (1997). The Psychophysics Toolbox. Spatial Vision, 10, 433-436.

Chun, M. M., \& Potter, M. C. (1995). A two-stage model for multiple target detection in rapid serial visual presentation. Journal of Experimental Psychology: Human Perception \& Performance, 21, 109-127.

Conci, M., Müller, H. J., \& Elliott, M. A. (2007). Closure of salient regions determines search for a collinear target configuration. Perception \& Psychophysics, 69, 32-47.

Drew, T., \& Shapiro, K. L. (2006). Representational masking and the attentional blink. Visual Cognition, 13, 513-528.

Driver, J., \& BAYLIS, C. G. (1989). Movement and visual attention: The spotlight metaphor breaks down. Journal of Experimental Psychology: Human Perception \& Performance, 15, 448-456.

DunCAN, J. (1984). Selective attention and the organization of visual information. Journal of Experimental Psychology: General, 113, 501-517

Egly, R., Driver, J., \& Rafal, R. D. (1994). Shifting visual attention between objects and locations: Evidence from normal and parietal lesion subjects. Journal of Experimental Psychology: General, 123, 161-177.

Haimson, C., \& Behrmann, M. (2001). Cued visual attention does not distinguish between occluded and occluding objects. Psychonomic Bulletin \& Review, 8, 496-503.

Hommel, B., Kessler, K., Schmitz, F., Gross, J., Akyürek, E., ShaPIRO, K., \& SCHNitZler, A. (2006). How the brain blinks: Towards a neurocognitive model of the attentional blink. Psychological Research, 70, 425-435.

Houghton, G., \& TipPer, S. E. (1994). A model of inhibitory mechanisms of selective attention. In D. Dagenbach \& T. H. Carr (Eds.), Inhibitory processes in attention, memory, and language (pp. 53-113). San Diego: Academic Press.

Jefferies, L. N., Ghorashi, S., Kawahara, J.-I., \& Di Lollo, V. (2007). Ignorance is bliss: The role of observer expectation in dynamic spatial tuning of the attentional focus. Perception \& Psychophysics, 69, 1162-1174.

JiANG, Y., \& ChUN, M. M. (2001). The influence of temporal selection on spatial selection and distractor interference: An attentional blink study. Journal of Experimental Psychology: Human Perception \& Performance, 27, 664-679.

JoRDAN, H., \& TiPPER, S. P. (1999). Spread of inhibition across an objects' surface. British Journal of Psychology, 90, 495-507.

Juola, J. F., Botella, J., \& Palacios, A. (2004). Task- and locationswitching effects on visual attention. Perception \& Psychophysics, 66, 1303-1317.

Kahneman, D., \& Treisman, A. (1984). Changing views of attention and automaticity. In R. Parasuraman \& D. A. Davies (Eds.), Varieties of attention (pp. 29-61). New York: Academic Press.

Kawahara, J.-I., \& Yamada, Y. (2006). Two noncontiguous locations can be attended concurrently: Evidence from the attentional blink. Psychonomic Bulletin \& Review, 13, 594-599.

Kellie, F. J., \& Shapiro, K. L. (2004). Object file continuity predicts attentional blink magnitude. Perception \& Psychophysics, 66, 692-712.

Kim, C.-Y., \& Blake, R. (2005). Psychophysical magic: Rendering the visible "invisible." Trends in Cognitive Sciences, 9, 381-388.

KLeIN, R. M., \& DicK, B. (2002). Temporal dynamics of reflexive attention shifts: A dual-stream rapid serial visual presentation exploration. Psychological Science, 13, 176-179.

Kristjánsson, A., \& NAKAYAMA, K. (2002). The attentional blink in space and time. Vision Research, 42, 2039-2050.

Lamy, D., \& EgETH, H. (2002). Object-based selection: The role of attentional shifts. Perception \& Psychophysics, 64, 52-66.
List, A., \& Robertson, L. C. (2007). Inhibition of return and objectbased attentional selection. Journal of Experimental Psychology: Human Perception \& Performance, 33, 1322-1334.

Moore, C. M., \& Fulton, C. (2005). The spread of attention to hidden portions of occluded surfaces. Psychonomic Bulletin \& Review, 12, 301-306.

Moore, C. M., Yantis, S., \& Vaughan, B. (1998). Object-based visual selection: Evidence from perceptual completion. Psychological Science, 9, 104-110.

O'Grady, R. B., \& MüLler, H. J. (2000). Object-based selection operates on a grouped array of locations. Perception \& Psychophysics, 62, 1655-1667.

Olivers, C. N. L., \& Meeter, M. (2008). A boost and bounce theory of temporal attention. Psychological Review, 115, 836-863.

Palmer, S. E. (1992). Common region: A new principle of perceptual grouping. Cognitive Psychology, 24, 436-447.

PALMER, S. [E.], \& Rock, I. (1994). Rethinking perceptual organization: The role of uniform connectedness. Psychonomic Bulletin \& Review, 1, 29-55.

PeLLI, D. G. (1997). The VideoToolbox software for visual psychophysics: Transforming numbers into movies. Spatial Vision, 10, 437-442.

Potter, M. C., Staub, A., \& O'Connor, D. H. (2002). The time course of competition for attention: Attention is initially labile. Journal of Experimental Psychology: Human Perception \& Performance, 28, 1149-1162.

RAYMOND, J. E. (2003). New objects, not new features, trigger the attentional blink. Psychological Science, 14, 54-59.

Raymond, J. E., Shapiro, K. L., \& Arnell, K. M. (1992). Temporary suppression of visual processing in an RSVP task: An attentional blink? Journal of Experimental Psychology: Human Perception \& Performance, 18, 849-860.

Shapiro, K. L., Raymond, J. E., \& Arnell, K. M. (1997). The attentional blink. Trends in Cognitive Sciences, 1, 291-296.

SHIH, S.-I. (2000). Recall of two visual targets embedded in RSVP streams of distractors depends on their temporal and spatial relationship. Perception \& Psychophysics, 62, 1348-1355.

TipPer, S. P., Driver, J., \& Weaver, B. (1991). Object-centred inhibition of return of visual attention. Quarterly Journal of Experimental Psychology, 43A, 289-298.

Vecera, S. P., \& FARAH, M. J. (1994). Does visual attention select objects or locations? Journal of Experimental Psychology: General, 123, 146-160.

Visser, T. A. W., Bischof, W. F., \& Di Lollo, V. (1999). Attentional switching in spatial and nonspatial domains: Evidence from the attentional blink. Psychological Bulletin, 125, 458-469.

Visser, T. A. W., Zuvic, S. M., Bischof, W. F., \& Di Lollo, V. (1999). The attentional blink with targets in different spatial locations. Psychonomic Bulletin \& Review, 6, 432-436.

Ward, R., Duncan, J., \& Shapiro, K. (1997). Effects of similarity, difficulty, and nontarget presentation on the time course of visual attention. Perception \& Psychophysics, 59, 593-600.

Watson, S. E., \& Kramer, A. F. (1999). Object-based visual selective attention and perceptual organization. Perception \& Psychophysics, 61, 31-49.

Yamada, Y., \& KaWAhara, J.-I. (2007). Dividing attention between two different categories and locations in rapid serial visual presentations. Perception \& Psychophysics, 69, 1218-1229.

\section{NOTE}

1. Note that, if task demands differ for $\mathrm{T} 1$ and $\mathrm{T} 2$ (e.g., a size discrimination task for $\mathrm{T} 1$ and a detection task for $\mathrm{T} 2$ ), a reliable $\mathrm{AB}$ is also found for repetitions of the target identity and location (Drew \& Shapiro, 2006; Ward, Duncan, \& Shapiro, 1997). Thus, task switches might lead to effects comparable to those of location switches (see Visser, Bischof, \& Di Lollo, 1999).

(Manuscript received July 21, 2008; revision accepted for publication July 14, 2009.) 